\title{
Localized anisotropic superconductors
}

\author{
A G Rojo $\nmid$ and C A Balseiroł \\ $\dagger$ Department of Physics, University of Michigan, Ann Arbor, MI 48109-1120, USA \\ $\ddagger$ Centro Atómico Bariloche and Instituto Balseiro, 8400-Bariloche, Argentina \\ Received 12 May 1998, in final form 9 July 1998
}

\begin{abstract}
We address the question of whether an anisotropic gap of $\mathrm{d}_{x^{2}-y^{2}}$ symmetry is compatible with localized states in the normal phase. The issue is important for high- $T_{c}$ superconductors for which a superconductor-to-insulator transition is observed, together with a number of experiments that support $d$-wave pairing. We prove that d-wave superconductivity is compatible with a localized normal state. When the coherence length is of the order of the lattice constant, the effects of localization are important. We find a re-entrant behaviour of superconductivity in the strongly disordered phase.
\end{abstract}

There is a growing body of experimental evidence for high- $T_{c}$ superconductors that indicates that the pairing state is of $\mathrm{d}_{x^{2}-y^{2}}$ symmetry [1]. For superconductors with an anisotropic order parameter, both magnetic and non-magnetic impurities are pair breaking. For d-wave symmetry, the effect of non-magnetic impurities is equivalent to that of magnetic impurities in s-wave superconductors [2]. Perturbation theory for the impurity scattering introduces a mean free path $\ell$ for the extended states, and the standard treatment indicates that anisotropic superconductivity is destroyed when $\xi_{0} / \ell=1 / \pi$ [3], with $\xi_{0}$ the coherence length. On the other hand, the charge dynamics for oxide superconductors is basically two dimensional, and it is known from the scaling theory of localization that in two dimensions all one-particle states are localized [4]. This conclusion is unchanged by the presence of electron-electron interaction $[4,5]$. In fact, the experimental evidence from resistivity measurements for low levels of doping is consistent with a divergent resistivity as $T \rightarrow 0$ that is cut off by the superconducting transition at $T=T_{c}$ [6]. The resistivity shows an upturn at a characteristic temperature $T_{\min }$ that is apparent when $T_{c}<T_{\min }$. Qualitatively, $T_{\min }$ corresponds to the temperature scale for which the inelastic scattering length is comparable to the localization length. Conversely, if the elastic mean free path is much bigger than the coherence length, the localization effects are not important, and the variation of $T_{c}$ with disorder will be given by the usual pair-breaking expressions. For the regime with $T_{c}<T_{\min }$, it is clear that superconductivity becomes established at a temperature low enough for the effects of localization to dominate the normal-state transport properties.

The purpose of the present work is to present a treatment of anisotropic superconductivity that incorporates the fact that the states from which the superconducting state is built up are localized, and reconcile two seemingly conflicting properties: the observed insulator-superconductor transition, and anisotropic pairing. We show that, if $\xi_{0} / a \gg 1$, with $a$ being the lattice constant, superconductivity is destroyed for small values of the disorder, and the localization effects are not important. In this case the critical value of the disorder is such that $\xi_{0}=\ell / \pi \ll \lambda$, with $\lambda$ the localization length. If $\xi_{0} / a$ 
is of order unity - as is the case for the oxide superconductors-when disorder increases, localization effects play a role before superconductivity is destroyed by conventional pairbreaking scattering. In this case, the dependence of the critical temperature on the disorder deviates from the celebrated Abrikosov-Gor'kov-Maki (AGM) theory [3]. We discuss the cases of p-wave and d-wave superconductivity.

For concreteness we consider fermions on a lattice described by the following Hamiltonian:

$$
H=H_{0}-U \sum_{x} \hat{\Delta}_{x}^{\dagger} \hat{\Delta}_{x}
$$

with $H_{0}$ being a one-electron Hamiltonian that includes disorder, with eigenstates $\varphi_{v}(x)$ and eigenvalues $\varepsilon_{\nu}$. The second term in (1) corresponds to an instantaneous attractive interaction with an implicit cut-off at a characteristic energy $\omega_{D}$. In order to model $\mathrm{d}_{x^{2}-y^{2}}$ symmetry, we choose $\hat{\Delta}_{x}^{\dagger}$ to be of the form

$$
\hat{\Delta}_{x}^{\dagger}=\frac{1}{\sqrt{2}} \sum_{\delta} \epsilon_{\delta}\left(c_{x \uparrow}^{\dagger} c_{x+\delta \downarrow}^{\dagger}-c_{x \downarrow}^{\dagger} c_{x+\delta \uparrow}^{\dagger}\right)
$$

with $\delta= \pm e_{1}, \pm e_{2}$ being the lattice vectors, and $\epsilon_{ \pm e_{1}}=-\epsilon_{ \pm e_{2}}=1$. We argue below that the effects of localization on the critical temperature $T_{c}$ for $\mathrm{d}$-wave pairing are qualitatively the same as those for p-wave pairing, an example of which is the triplet pairing [7], that we model with

$$
\hat{\Delta}_{x \sigma}^{\dagger}=\sum_{\delta} \epsilon_{\delta}^{\prime} c_{x \sigma}^{\dagger} c_{x+\delta \sigma}^{\dagger}
$$

where $\epsilon_{+e_{1}}=-\epsilon_{-e_{1}}=1$ and $\epsilon_{+e_{2}}=\epsilon_{-e_{2}}=0$.

The critical temperature is determined by the self-consistent solution of the following linearized gap equation [8]:

$$
\Delta_{x}=\sum_{x^{\prime}} K\left(x, x^{\prime}\right) \Delta_{x^{\prime}}
$$

where $\Delta_{x}=\left\langle\hat{\Delta}_{x}\right\rangle$, and the kernel $K$, written in terms of the exact eigenstates of $H_{0}$, is given by

$$
K\left(x, x^{\prime}\right)=U \frac{T}{2} \sum_{\omega_{n}} \sum_{\mu \nu \delta \delta^{\prime}} \epsilon_{\delta} \epsilon_{\delta^{\prime}} \frac{\varphi_{\mu}^{*}(x) \varphi_{v}^{*}(x+\delta) \varphi_{v}\left(x^{\prime}\right) \varphi_{\mu}\left(x^{\prime}+\delta\right)}{\left(\varepsilon_{v}-\mathrm{i} \omega_{n}\right)\left(\varepsilon_{\mu}+\mathrm{i} \omega_{n}\right)}
$$

with $T$ the temperature and $\omega_{n}=(2 n+1) \pi T$ the Matsubara frequencies. Also, we have taken $\hbar=k_{B}=1$. From now on we will take the eigenstates as real.

We next assume that the gap is uniform, $\Delta_{x}=|\Delta|$, which is justified for $\omega_{D} \gg \Delta W$, with $\Delta W$ the typical level spacing between states within a localization length of each other. In that case we can integrate (4) over $x$ and $x^{\prime}$ and reach the condition

$$
1=\frac{T}{2} N_{F} U \int \mathrm{d} \xi \mathrm{d} \xi^{\prime} \sum_{\omega_{n}} \frac{g\left(\xi-\xi^{\prime}\right)}{\left(\xi^{\prime}+\mathrm{i} \omega_{n}\right)\left(\xi-\mathrm{i} \omega_{n}\right)}
$$

with $N_{F}$ the density of states at the Fermi level, and $g(\omega)$ is the power spectrum of the operator

$$
\hat{D}=\sum_{x, \delta} \epsilon_{\delta}(|x+\delta\rangle\langle x|+| x\rangle\langle x+\delta|)
$$

given by

$$
g(\omega)=\sum_{\nu} \overline{|\langle\mu|\hat{D}| \nu\rangle|^{2}} \delta\left(\varepsilon_{v}-\varepsilon_{F}-\omega\right)
$$


where the line over the square of the matrix element indicates an average over states $\mu$ such that $\varepsilon_{\mu}=\varepsilon_{F}$. For s-wave symmetry, $\hat{D}$ corresponds to the density operator

$$
\hat{D}=\sum_{x}|x\rangle\langle x|
$$

and $g(\omega)=\delta(\omega)$, since the density response is not sensitive to scattering (in the $q=0$ limit, which is the case in which we are interested). This is valid even when the states $\varphi_{v}(x)$ are localized. With this frequency dependence of $g(\omega)$, one obtains an equation for the critical temperature identical to that of the pure system. This is the extension of the Anderson theorem to the case of localized states, which was discussed by Ma and Lee using a variational approach [9]. We conclude that under the above assumptions $\left(\omega_{D} \gg \Delta W\right.$ and a uniform gap), the effects of localization on the critical temperature are contained, through equation (5), in the frequency dependence of the spectral function of the operator that has the symmetry of the order parameter. The function $g(\omega)$ can be calculated diagrammatically, since it is given by a two-particle bubble with bare vertices

$$
\gamma_{k}^{d}=\cos k_{x}-\cos k_{y}
$$

for d-wave symmetry, and

$$
\gamma_{k}^{p}=\mathrm{i} \sin k_{x}
$$

for p-wave symmetry. From now on we ignore the lattice effects, and take

$$
\begin{aligned}
\gamma_{k}^{d} & =\cos 2 \theta_{k} \\
\gamma_{k}^{p} & =\cos \theta_{k}
\end{aligned}
$$

which corresponds to a gap function

$$
\Delta(\boldsymbol{k})=\Delta(T) \gamma_{k}^{i} \quad(i=\mathrm{p}, \mathrm{d}) .
$$

We first write equation (6) as

$$
g_{i}(\omega)=\frac{1}{2 \pi^{2}} \operatorname{Re} \sum_{k, k^{\prime}} \gamma_{k}^{i} \Phi_{k, k^{\prime}}(\omega) \gamma_{k^{\prime}}^{i} \quad(i=\mathrm{p}, \mathrm{d})
$$

with

$$
\Phi_{\boldsymbol{k}, \boldsymbol{k}^{\prime}}=\left\langle G^{\mathrm{R}}\left(\boldsymbol{k}, \boldsymbol{k}^{\prime} ; \varepsilon_{F}\right) G^{\mathrm{A}}\left(\boldsymbol{k}^{\prime}, \boldsymbol{k}, \boldsymbol{\varepsilon}_{F}^{+} \omega\right)\right\rangle
$$

where now $\langle\cdots\rangle$ denotes impurity averaging, and $G^{\mathrm{R}}$ and $G^{\mathrm{A}}$ indicate the retarded and advanced one-particle Green functions. We follow the work of Vollhardt and Wölfle (VW) [10], and compute $g(\omega)$ within the self-consistent theory of localization. We prove that the frequency dependence of (9) is essentially the same as that of the conductivity. The quantity $\Phi_{k, k^{\prime}}$ obeys the Bethe-Salpeter equation

$$
\Phi_{k, k^{\prime}}(\omega)=G_{k}^{\mathrm{R}} G_{k}^{\mathrm{A}} \delta_{k, k^{\prime}}+G_{k}^{\mathrm{R}} G_{k}^{\mathrm{A}} \sum_{k^{\prime \prime}} U_{k, k^{\prime \prime}}(\omega) \Phi_{k^{\prime \prime} k^{\prime}}(\omega)
$$

with $U_{k, k^{\prime \prime}}(\omega)$ the irreducible vertex function. In Anderson localization, single-particle quantities (e.g. the density of states) are smoothly varying functions of the disorder. It is then reasonable to approximate the self-energy $\Sigma$ by the lowest-order result in the impurity scattering $U_{0}$, and use the Green's functions in the form

$$
G_{k}^{\mathrm{R}}(\varepsilon)=\frac{1}{\varepsilon-\varepsilon_{k}+\mathrm{i} / 2 \tau}
$$


with $1 / \tau=2 \pi N_{F} U_{0}$, and $G^{\mathrm{R}}=\left(G^{\mathrm{A}}\right)^{*}$. We have assumed a $\delta$-correlated disordered potential $u(x)$, such that

$$
\langle u(0) u(x)\rangle=u_{0}^{2} n_{\mathrm{imp}} \delta(x)=U_{0} \delta(x) .
$$

Using this expression for the Green's functions, we can write the Bethe-Salpeter equation as a kinetic equation in the form

$$
\left(\omega-\frac{\mathrm{i}}{\tau}\right) \Phi_{k, k^{\prime}}=-\Delta G_{k}\left[\delta_{k, k^{\prime}}+\sum_{k^{\prime \prime}} U_{k, k^{\prime \prime}} \Phi_{k^{\prime \prime} k^{\prime}}\right]
$$

with $\Delta G_{k} \equiv G_{k}^{\mathrm{R}}-G_{k}^{\mathrm{A}}$. If we replace in (13) the irreducible vertex by the bare vertex $U_{0}$, we obtain

$$
g_{i}(\omega)=\frac{1}{2} \frac{1}{\pi} \frac{\tau}{1+(\omega \tau)^{2}}
$$

where the prefactor $1 / 2$ comes from the angular average of the squared vertices which is the same for the p- and d-wave cases (this prefactor would be one for s waves).

For the p-wave case, the function $g_{i}(\omega)$ has the same structure of the conductivity, and the above equation corresponds to the Drude formula. For the d-wave case, $g_{d}(\omega)$ was obtained earlier in a treatment of the Raman response in the $l=2$ channel in the presence of impurities [11]. However, since the Raman response $R(\omega)$ is given by a correlation function, there is an additional factor of $\omega$ and $R(\omega)=\omega g_{d}(\omega)$. Inserting (14) in (5), we obtain the well known expression for the critical temperature variation [12]:

$$
-\ln \left(T_{c} / T_{c 0}\right)=\psi\left(1 / 2+1 / 4 \pi \tau T_{c}\right)-\psi(1 / 2) .
$$

To account for the effects of localization, the Bethe-Salpeter equation has to be solved to higher order. Since in this regime there are some differences between the p-wave $(l=1$ channel) and d-wave ( $l=2$ channel) cases, we discuss them separately.

(i) The p-wave case $(\boldsymbol{l}=\mathbf{1})$. The low-frequency $l=1 \mathrm{kernel}$ is the same (except for a prefactor) as the current relaxation kernel. The result for $g_{p}(\omega)$ is identical to the expression obtained by $\mathrm{VW}$, and is given by

$$
g_{p}(\omega)=\frac{1}{4 \pi} \frac{\tau}{1+(\widetilde{\omega} \tau)^{2}}
$$

with $\widetilde{\omega}=\omega+\omega_{0}^{2} / \omega$. The characteristic frequency $\omega_{0}$ is finite in the localized phase, and is given by

$$
\omega_{0}=v_{F} /(\sqrt{2} \lambda)
$$

with $\lambda$ the localization length.

From this consideration, one can compute an expression for the relative change of the critical temperature with disorder, which extends the AGM theory for the case of localized anisotropic superconductors. Our result is then

$$
\begin{aligned}
\ln \left(\frac{T_{c 0}}{T_{c}}\right)=- & \ln \left(\frac{T_{c 0}}{\omega_{D}}\right) \frac{2 \tau}{\tau^{-}}+\frac{\tau}{\tau^{+}}\left[\psi\left(\frac{1}{2}+\rho_{+}\right)-\psi\left(\frac{1}{2}\right)\right] \\
& -\frac{\tau}{\tau^{-}}\left[\psi\left(\frac{1}{2}+\rho_{-}\right)-\psi\left(\frac{1}{2}\right)\right]
\end{aligned}
$$

where

$$
1 / \tau^{ \pm}=\sqrt{\omega_{0}^{2}+(1 / 2 \tau)^{2}} \pm 1 / 2 \tau \quad \rho_{ \pm}=1 / 4 \pi \tau^{ \pm} T_{c} .
$$




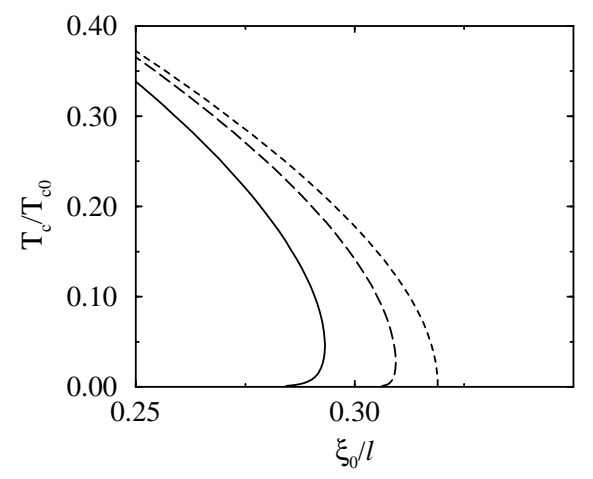

Figure 1. The relative critical temperature $T_{c} / T_{c 0}$ for the p-wave case versus $\xi_{0} / \ell$ for $k_{F} \xi_{0}=10$ (short-dashed line), 1.0 (dashed line), 0.75 (solid line), and $\omega_{D} / T_{c 0}=0.1$. The AbrikosovGor'kov-Maki curve is indistinguishable from the one corresponding to $k_{F} \xi_{0}=10$.

The above expression gives the change in critical temperature as a function of $\tau$ and $\omega_{0}$. In contrast with the case for the AGM formula, in our case the relative change in $T_{c}$ is dependent of the cut-off frequency $\omega_{D}$. The self-consistent theory of localization provides a relation between the two relevant parameters $\omega_{0}$ and $\tau$ introduced by potential scattering:

$$
\omega_{0} \tau=\ell / \sqrt{2} \lambda \quad \frac{\lambda}{\ell}=\left(\mathrm{e}^{\pi k_{F} \ell / 2}-1\right)^{1 / 2} .
$$

Consequently, the relative change in the critical temperature is given by the three independent dimensionless parameters $\omega_{D} / T_{c 0}, k_{F} \xi_{0}$, and $k_{F} \ell$ (see figure 1). The effects of localization are important when $k_{f} \xi_{0}$ is of order one. In this regime the results deviate from the AGM theory; in particular $T_{c} / T_{c 0}$ shows a re-entrant behaviour.

We now discuss briefly the mechanism underlying the re-entrant behaviour in $T_{c}$. The re-entrance to a normal state as temperature decreases can occur if the entropy of the superconducting phase is higher than the entropy of the normal state at very low temperatures. This can happen if the localization length is of the order of the mean free path. In this regime one can estimate, for the p-wave case, the density of states $\rho(E)$ of the quasiparticles by computing the quasiparticle energies $E_{v}$ as a correction of the particle energies $\varepsilon_{v}$ in second order in the pairing interaction [8]:

$$
\rho(E)=N_{0} /\left|\frac{\partial E}{\partial \varepsilon}\right|=N_{0} /\left(1+\left.\Delta^{2} \frac{\partial}{\partial \varepsilon} \int \mathrm{d} \omega \frac{g(\omega)}{2 \varepsilon+\omega}\right|_{\varepsilon=E}\right) .
$$

The result is then that the density of states at the Fermi energy can be bigger than the normalstate density of states, and the superconducting entropy is higher at low temperatures. The behaviour of $\rho(E)$ is non-monotonic in $E$ and one gets two transitions as the temperature increases. Although the range in values of the disorder for which this re-entrant behaviour is visible is small, one expects the quasiparticle density of states to have a structure with a maximum at the chemical potential for a wider range of disorder.

(ii) The d-wave case $(\boldsymbol{l}=\mathbf{2})$. The correlation functions $g_{i}(\omega)$ for the $\mathrm{p}$ and $\mathrm{d}$ channels have a similar structure. However, since the d-wave operator does not correspond to a conserved current, there is in this case an additional singular contribution that gives rise to a term $\propto \delta(\omega)$. We see from equation (6) that the diagonal terms $\langle v|\hat{D}| v\rangle$, which are identically zero for $l=1$, are non-zero when $\hat{D}$ has d-wave symmetry. Physically, the weight of the delta function corresponds to the fraction of Cooper pairs formed with electrons occupying 
the same localized state. A precise computation of this weight requires knowledge of the structure of the localized wave functions, and we estimate it in the following form. The singular contribution is of the form $S_{d} \delta(\omega)$, with $S_{d}$ the d-wave component of the wave function:

$$
S_{d} \sim \int r \mathrm{~d} r \mathrm{~d} \theta \Psi^{2} \cos 2 \theta .
$$

If we approximate $\Psi^{2}$ as a Gaussian random variable located at each site, and distributed over the localization length $\lambda, S_{d}$ is of the order of the fluctuation of the sum over the random variables. The number of sites over which the function is non-zero is $\sim \lambda^{2} / a^{2}$, with $a$ the lattice constant, and we obtain $S_{d} \sim 1 / \lambda^{2}$. This is only an estimate, and in general we will show that the correlation function of equation (6) will be given by

$$
g_{d}(\omega)=\left(1-S_{d}\right) \frac{1}{2 \pi} \frac{\tau_{d}}{1+\left(\widetilde{\omega} \tau_{d}\right)^{2}}+\frac{S_{d}}{2} \delta(\omega)
$$

where the prefactor $\left(1-S_{d}\right)$ guarantees that the $g_{d}(\omega)$ satisfies the sum rule

$$
\int_{0}^{\infty} \mathrm{d} \omega g_{d}(\omega)=\frac{1}{2}
$$

which can be proven immediately by integrating equation (6).

For the computation of the finite-frequency contribution of $g_{d}(\omega)$ we start from equation (13). Following VW, we observe that since $\Delta G_{k}$ is strongly peaked at the Fermi momentum $k=k_{F}$, the dependence of $\Phi_{k, k^{\prime}}$ on the magnitude of the wave vectors will be dominated by $\Delta G_{k}$. We define

$$
\Phi_{k}=\sum_{k^{\prime}} \Phi_{k, k^{\prime}} \cos 2 \theta_{k^{\prime}}
$$

and extract the angular dependence on $k$ using a Legendre expansion in which we keep terms up to the $l=2$ one:

$$
\Phi_{k}=\frac{\Delta G_{k}}{-2 \pi \mathrm{i} N_{F}} \sum_{k^{\prime}}\left[1+2 \cos \theta_{k} \cos \theta_{k^{\prime}}+2 \cos 2 \theta_{k} \cos 2 \theta_{k^{\prime}}\right] \Phi_{k^{\prime}} .
$$

Multiplying (13) by $\cos 2 \theta_{\boldsymbol{k}} \cos 2 \theta_{\boldsymbol{k}^{\prime}}$, summing over $\boldsymbol{k}, \boldsymbol{k}^{\prime}$, and using (21), we obtain

$$
\sum_{k, k^{\prime}} \cos 2 \theta_{k} \Phi_{k, k^{\prime}} \cos 2 \theta_{k^{\prime}}=\frac{-\mathrm{i} \pi N_{F}}{\omega-M(\omega)}
$$

where $M(\omega)$ is a ' $l=2$ relaxation kernel', given by

$$
M(\omega)=\frac{\mathrm{i}}{\tau}+\frac{\mathrm{i}}{\pi N_{F}} \sum_{k, k^{\prime}} \cos 2 \theta_{k} \Delta G_{k} U_{k, k^{\prime}} \Delta G_{k^{\prime}} \cos 2 \theta_{k^{\prime}} .
$$

The structure of $M(\omega)$ is very similar to that of the current relaxation kernel. Note that in deriving this expression we have neglected terms that mix different angular dependences in $M(\omega)$, and that give rise to factors $\cos m \theta_{k} \cos 2 \theta_{k^{\prime}}$, with $m=0,1,2$. These terms do not appear in the treatment of VW. Neglecting these terms is justified, since we are anticipating the inclusion of the contribution to the irreducible vertex that is the origin of the divergence of $M(\omega)$. The infrared divergence of $M$ comes from the maximally crossed (MC) diagrams [13], which are irreducible, and contribute with

$$
U_{\boldsymbol{k}, \boldsymbol{k}^{\prime}}^{\mathrm{MC}}=\frac{\mathrm{i} U_{0} / \tau}{\omega+\mathrm{i} D_{0}\left(\boldsymbol{k}+\boldsymbol{k}^{\prime}\right)^{2}}
$$


for $\boldsymbol{k} \simeq-\boldsymbol{k}^{\prime}$, and with $D_{0}$ the bare diffusion constant. Due to this divergence we can take $k=\boldsymbol{k}^{\prime}$ for the angular integral and the 'off-diagonal' contributions vanish due to orthogonality. Using the above $U_{k, k^{\prime}}^{\mathrm{MC}}$ in (23), we obtain the logarithmic lowfrequency divergence $M(\omega) \sim \log \omega$ that is familiar from the perturbation theory of the conductivity [14].

The low-frequency kernel is the same (except for a prefactor) as the current relaxation kernel, and it will still be related to the diffusion constant

$$
D(\omega)=\mathrm{i} D_{0}[M(\omega) \tau]^{-1} .
$$

This allows us to go beyond perturbation theory and determine $M(\omega)$ self-consistently through the equation

$$
M(\omega)=\frac{\mathrm{i}}{\tau}-\frac{2}{\tau} \sum_{k} \frac{1}{\omega-k^{2} D_{0}[M(\omega) \tau]^{-1}} .
$$

Equation (25) can be solved for low frequencies, giving

$$
M(\omega)=\frac{\mathrm{i}}{\tau}-\frac{\omega_{0}^{2}}{\omega}
$$

and

$$
g_{d}(\omega>0)=\frac{N_{F}}{2 \pi} \frac{\tau}{1+(\widetilde{\omega} \tau)^{2}}
$$

which implies the following equation for the critical temperature:

$$
\begin{aligned}
\ln \left(\frac{T_{c 0}}{T_{c}}\right)=D^{-1} & \left\{-\ln \left(\frac{T_{c 0}}{\omega_{D}}\right) \frac{2 \tau}{\tau^{-}}+\frac{\tau}{\tau^{+}}\left[\psi\left(\frac{1}{2}+\rho_{+}\right)-\psi\left(\frac{1}{2}\right)\right]\right. \\
& \left.-\frac{\tau}{\tau^{-}}\left[\psi\left(\frac{1}{2}+\rho_{-}\right)-\psi\left(\frac{1}{2}\right)\right]\right\}
\end{aligned}
$$

with

$$
D=1+\frac{S_{d} \tau}{1-S_{d}} \sqrt{\omega_{0}^{2}+(1 / 2 \tau)^{2}}
$$

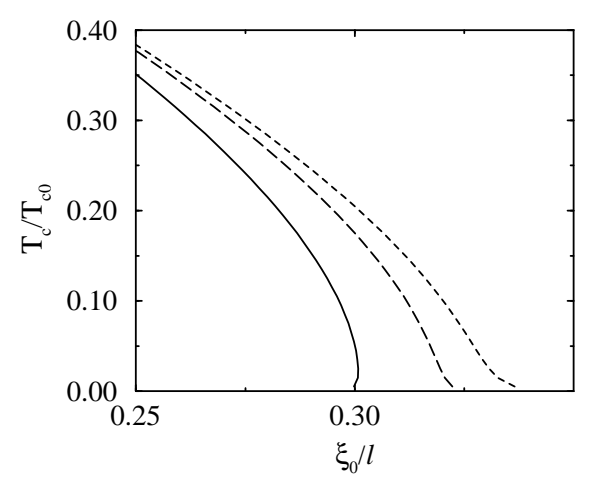

Figure 2. The relative critical temperature $T_{c} / T_{c 0}$ for the d-wave case versus $\xi_{0} / \ell$ for $k_{F} \xi_{0}=10$ (short-dashed line), 1.0 (dashed line), 0.75 (solid line), and $\omega_{D} / T_{c 0}=0.1$. The plots correspond to $S_{d}=0.2$.

Irrespectively of the value of $k_{F} \xi_{0}$, if $k_{F} \ell \gg 1$ (small disorder), the states are essentially extended, and the relative change in $T_{c}$ is given by the AGM theory. The effects of 
localization can be apparent when $k_{F} \xi_{0}$ is of order one, as is the case for the oxide superconductors. For $\xi_{0} / l>1 / \pi$ the critical temperature is no longer zero as in the case of the AGM theory, but has an exponentially small value (see figure 2). In this regime, the superconducting condensate wave function is constructed from localized oneparticle states for which the localization length is only one order of magnitude larger than the coherence length. For large enough disorder, our approximation breaks down, since the order parameter is no longer uniform, and the critical temperature should go to zero. The possibility of constructing a superconducting condensate from one-particle sates with relatively small localization lengths has been analysed in the case of s-wave superconductors [9], for which the Anderson theorem holds. Our results show that, in the case of p- or dwave symmetries, where the potential scattering is pair breaking, superconductivity and localization can also coexist.

To conclude we comment on the following points.

(i) If disorder is too strong, the hypothesis made above of a uniform gap does not hold. For all of the curves of figure 1 , the inequality $N_{F} \lambda^{2} \Delta_{0}>1$ is satisfied, which guarantees that spatial fluctuations in the gap are negligible [9].

(ii) An important effect on $T_{c}$ in s-wave superconductors is due to the enhancement of the effective screened Coulomb repulsion due to disorder [15]. For d-wave symmetry the short-range Coulomb repulsion does not affect the effective pairing, and consequently the effects of the Coulomb interaction are not as important. In particular, our results are not changed at all by a local Coulomb repulsion, which corresponds to Hubbard-type models used to describe the copper oxide planes.

(iii) As regards experiments on high- $T_{c}$ oxides, the superconducting-insulating transition has been induced by various mechanisms: irradiation [16], ion substitution [17], or doping [6]. In comparing our results with experiments, one should be able to isolate the effects of hole doping and the effects of disorder.

In summary, we have shown that p- and d-wave superconductivity are compatible with Anderson localization of the one-particle states. Our theory distinguishes between the two symmetries in the localized regime, and gives essentially identical results in the extended phase. In addition, out treatment extends previous calculations of the Raman response in disordered systems to the localized phase.

\section{Acknowledgments}

This work was supported by the National Science Foundation under grant INT-9602962, and by the CONICET, Argentina. We thank Eduardo Fradkin, J Rasul, P A Lee, D Vollhardt, and $\mathrm{C} \mathrm{M}$ Varma for discussions.

\section{References}

[1] Wollman D A, Van Harlingen D J, Lee W C, Ginsberg D M and Leggett A J 1993 Phys. Rev. Lett. 712134 Tsuei C C, Kirtley J R, Chi C C, Yu-Jahnes L S, Gupta A, Shaw T, Sun J Z and Ketchen M B 1994 Phys. Rev. Lett. 73593

[2] Markowitz D and Kadanoff L P 1993 Phys. Rev. 131563

Tsuneto T 1962 Prog. Theor. Phys. 28857

[3] Abrikosov A A and Gor'kov L P 1960 Zh. Eksp. Teor. Fiz. 391781 (Engl. Transl. 1961 Sov. Phys.-JETP 12 1243)

Maki K 1969 Superconductivity vol 2, ed R D Parks (New York: Dekker)

[4] Lee P A and Ramakrishnan T V 1985 Rev. Mod. Phys. 57287 
[5] Belitz D and Kirkpatrick T R 1994 Rev. Mod. Phys. 66261

[6] Takagi H, Batlogg B, Kao H L, Kwo J, Cava R J, Krajewski J J and Peck W F Jr 1992 Phys. Rev. Lett. 69 2975

[7] Leggett A J 1975 Rev. Mod. Phys. 47331

[8] de Gennes P G 1966 Superconductivity of Metals and Alloys (New York: Benjamin)

[9] Ma M and Lee P A 1985 Phys. Rev. B 325658

[10] Vollhardt D and Wölfle P 1980 Phys. Rev. B 224666

Vollhardt D and Wölfle P 1992 Electronic Phase transitions ed W Hanke and Yu V Kopaev (Amsterdam: Elsiever)

[11] Zawadowski A and Cardona M 1990 Phys. Rev. B 4210732

[12] Radtke R J, Levin K, Schüttler H-B and Norman M R 1993 Phys. Rev. B 48653

[13] Langer J S and Neal T 1966 Phys. Rev. Lett. 16984

[14] Gor'kov L P, Larkin A I and Khmel'nitskii D E 1979 JETP Lett. 30228

[15] Anderson P W, Mutalib K A and Ramakrishnan T V 1983 Phys. Rev. B 28117

[16] Valles J M, White A E, Short K T, Dynes R C, Garno J P, Levi A F J, Anzlowar M and Baldwin K 1989 Phys. Rev. B 3911599

[17] Quitmann C, Almers P, Ma J, Kelley R J, Berger H, Cai X, Margaritondo G and Onellion M 1996 Phys. Rev. B 536819 